\title{
Current Status of Magnetic Resonance Imaging in Patients with Malignant Uterine Neoplasms: A Review
}

\author{
Yu-Ting Huang, $\mathrm{MD}^{1,2,4 *}$, Yen-Ling Huang, $\mathrm{MD}^{1,4 *}$, Koon-Kwan Ng, MD ${ }^{1,2,4}$, Gigin Lin, MD, PhD ${ }^{1,3,4}$ \\ ${ }^{1}$ Department of Medical Imaging and Intervention, Imaging Core Laboratory, Institute for Radiological Research, Chang Gung Memorial Hospital \\ at Linkou, Taoyuan, Taiwan; ${ }^{2}$ Department of Diagnostic Radiology, Chang Gung Memorial Hospital at Keelung, Keelung, Taiwan; ${ }^{3}$ Clinical \\ Metabolomic Core Laboratory, Chang Gung Memorial Hospital at Linkou, Chang Gung University, Taoyuan, Taiwan; ${ }^{4}$ Gynecologic Cancer Research \\ Center, Chang Gung Memorial Hospital, Chang Gung University College of Medicine, Taoyuan, Taiwan
}

In this study, we summarize the clinical role of magnetic resonance imaging (MRI) in the diagnosis of patients with malignant uterine neoplasms, including leiomyosarcoma, endometrial stromal sarcoma, adenosarcoma, uterine carcinosarcoma, and endometrial cancer, with emphasis on the challenges and disadvantages. MRI plays an essential role in patients with uterine malignancy, for the purpose of tumor detection, primary staging, and treatment planning. MRI has advanced in scope beyond the visualization of the many aspects of anatomical structures, including diffusion-weighted imaging, dynamic contrast enhancement-MRI, and magnetic resonance spectroscopy. Emerging technologies coupled with the use of artificial intelligence in MRI are expected to lead to progressive improvement in case management of malignant uterine neoplasms.

Keywords: Uterine neoplasm; Magnetic resonance imaging; Endometrial cancer; Sarcoma

\section{INTRODUCTION}

Malignant uterine neoplasms are reported as the most common malignancy in the pelvis of female patients $(1,2)$. The pathological origins of malignant uterine neoplasms derive from the stromal/mesenchymal layer (leiomyosarcoma [LMS], or endometrial stromal sarcoma [ESS]) or epithelial layer (endometrial cancer [EC], or uterine carcinosarcoma [UCS]). EC accounts for the majority of cases, whereas uterine sarcomas are far less common, with approximate rate of one in twelve of all uterine malignancies $(3,4)$.

Received February 8, 2018; accepted after revision July 17, 2018. This study was supported by Chang Gung Medical Foundation grant CPRPG3G0022, National Science Council (Taiwan) MOST 104-2314-B-182A-095-MY3, MOST 106-2314-B-182A-019-MY3. *These authors contributed equally to this work.

Corresponding author: Gigin Lin, MD, PhD, Department of Medical Imaging and Intervention, Imaging Core Laboratory, Institute for Radiological Research, Chang Gung Memorial Hospital at Linkou, 5 Fuhsing St., Guishan, Taoyuan 33382, Taiwan.

- Tel: (8863) 3281200 - Fax: (8863) 3971936

- E-mail: giginlin@cgmh.org.tw

This is an Open Access article distributed under the terms of the Creative Commons Attribution Non-Commercial License (https://creativecommons.org/licenses/by-nc/4.0) which permits unrestricted non-commercial use, distribution, and reproduction in any medium, provided the original work is properly cited.
Per National Comprehensive Cancer Network guideline, primary surgery is the recommended treatment for all the abovementioned malignant uterine neoplasms (5). In contrast, cervical cancer, is primarily treated using chemoradiation, with indication for surgery in stage IBIIA disease alone (5). The different clinical and therapeutic aspects of cervical cancers are beyond the scope of this article but are previously reported (6).

Robust imaging technology is continuously evolving to improve preoperative treatment planning. Magnetic resonance imaging (MRI) has superior performance than ultrasonography and computed tomography (CT) for detecting the myometrial invasion (7), with advanced capability beyond the visualization of different aspects of the anatomical structures. Diffusion-weighted imaging (DWI) measures the Brownian motion of molecules and highlights the increased cellularity of cancer tissue through quantitative evaluation using the apparent diffusion coefficient (ADC) map (8). Dynamic contrast enhancement (DCE)-MRI utilizes the intravenous gadolinium-based contrast agent which is contained in the healthy blood vessel by means of the functional tight-junction but leaks out through the process of neovascularization in the tumor (9), thus providing adequate model of the blood flow, 
extraction fraction, blood volume, volume of extravascular extracellular space, capillary permeability surface area product, and transfer from the blood to tissue. The magnetic resonance spectroscopy (MRS) technique enables semi-quantitative assessment of the chemical composition in selected region(s) of interest (10). Currently, most clinical magnetic resonance (MR) scanners have routine sequences for proton MRS measurement, providing a range of metabolic and functional information integrated with complementary MRI localization; however, MRS requires additional acquisition time for post processing, and has difficulty in shimming due to air and intestinal movement (11). The commonly used MR sequences are summarized in Table 1.

The aim of this review is to summarize experience and literature-based evidence on the clinical role of MRI in patients with uterine malignancies. We review common imaging characteristics of the uterine malignancies originating from various tumor types, with particular emphasis on the diagnostic challenges and disadvantages.

\section{Leiomyosarcoma}

\section{LMS: Clinicopathological Features and Diagnostic \\ Challenges \\ Leiomyosarcoma, a malignant smooth muscle tumor, is} the most common uterine sarcoma which occurs in patients with peri- or postmenopausal status or over 40 years of age $(3,4)$. Uterine smooth muscle tumor with uncertain malignant potential (STUMP), is a rare entity clinically considered to be of low malignant potential based on the possibility of recurrence. Malignancy should be suspected in case of occurrence of tumor growth in menopausal women not undergoing hormonal replacement therapy. In both LMS and STUMP, staging is the most significant prognostic factor; and in the tumor confined in the corpus, tumor size of less than $5 \mathrm{~cm}$ is associated with better survival rate $(3,4)$. Distinguishing between LMS, STUMP, and benign leiomyomas using MRI remains a challenge, especially due to overlapping of variable atypical imaging features (12). LMS, STUMP, and benign leiomyomas share similar clinical presentations including abnormal vaginal bleeding, palpable pelvic mass, and occasional pelvic pain, leading to approximately $0.5 \%$ of resected tumors with preoperative diagnosis of leiomyoma that are unexpectedly revealed as LMS based on final histopathology. However, the large uterine size and rapid tumor growth, are not associated with the increased risk of sarcoma $(13,14)$. Tissue diagnosis based on the endometrial sampling is challenging due to the intramuscular location of LMS and STUMP. In the era of minimal invasive surgery and conservative treatment, accurate preoperative imaging diagnosis of LMS plays a crucial role in selecting patient for non-surgical treatment (15).

\section{LMS: Radiological Advantages and Disadvantages}

MRI features of LMS include nodular borders, flow-void

Table 1. Acquisition Protocol of MRI for Uterine Malignancy

\begin{tabular}{|c|c|c|c|c|c|}
\hline & CE-MRI & DWI & T2WI & T1WI & MRS \\
\hline Pulse sequence & Turbo spin echo & $\begin{array}{l}\text { Single-shot } \\
\text { echo-planar }\end{array}$ & Turbo spin echo & Turbo spin echo & $\begin{array}{r}\text { Point-resolved } \\
\text { spectroscopy }\end{array}$ \\
\hline Orientation & Axial and sagittal & Axial and sagittal & Axial and sagittal & Axial & $\mathrm{N} / \mathrm{A}$ \\
\hline Slice thickness/gap (mm) & $4 / 1$ & $4 / 1$ & $4 / 1$ & $4 / 1$ & $12 \times 12 \times 12$ \\
\hline Repetition time (msec) & 567 & 3300 & 5630 & 626 & 2000 \\
\hline Echo time (msec) & 10 & 79 & 87 & 11 & 35 \\
\hline Field of view $(\mathrm{cm})$ & 20 & 30 & 20 & 20 & N/A \\
\hline $\begin{array}{l}\text { Acquisition matrix } \\
\text { (phase } x \text { frequency) }\end{array}$ & $256 \times 320$ & $128 \times 128$ & $256 \times 320$ & $256 \times 320$ & 1 \\
\hline Averages (NEX) & 2 & 4 & 3 & 2 & 128 \\
\hline Echo train length & 5 & 120 & 13 & 3 & 1 \\
\hline Flip angle & 150 & 180 & 150 & 150 & 90 \\
\hline GRAPPA factor & 2 & 2 & 2 & 2 & 1 \\
\hline Fat saturation & CHESS & CHESS & None & None & None \\
\hline Acquisition time (sec) & 185 & 63 & 176 & 133 & 256 \\
\hline
\end{tabular}

$\mathrm{CE}=$ contrast-enhanced, CHESS $=$ chemically selective suppression, DWI $=$ diffusion-weighted imaging $\left(b=0,1000 \mathrm{~s} / \mathrm{mm}^{2}\right), \mathrm{GRAPPA}=$ generalized auto-calibrating partially parallel acquisition, $M R I=$ magnetic resonance imaging, $M R S=$ magnetic resonance spectroscopy, $\mathrm{N} / \mathrm{A}=$ not applicable, NEX = number of excitations, T1WI $=\mathrm{T} 1$-weighted images, $\mathrm{T} 2 \mathrm{WI}=\mathrm{T} 2$-weighted images 
areas, T1 hyperintensity areas, and rapid enhancement at the early phase post-injection of contrast medium $(10,16$ 18); and shared features with that of uterine sarcomas include heterogeneous, intermediate T2 signal, and high signal on DWI with low ADC value, associated with intratumoral hemorrhage and necrosis (19-21). In contrast, benign uterine leiomyomas show low signal intensity relative to that of the myometrium on T2-weighted images (T2WI), well-defined pushing borders, and possible strong post-contrast enhancement $(22,23)$. Uterine leiomyomas usually demonstrate various types of degeneration, edema, and growth pattern; and their image findings may overlap with that of malignant tumors (12). Contrast-enhanced MRI has been proven to have superior performance than T2WI in detecting tumor necrosis (10), due to the shared feature of intratumoral T2 hyperintensity in benign leiomyomas with degeneration. Despite limited available data, central nonenhancement (CNE) and well-demarcated pocket-like non-

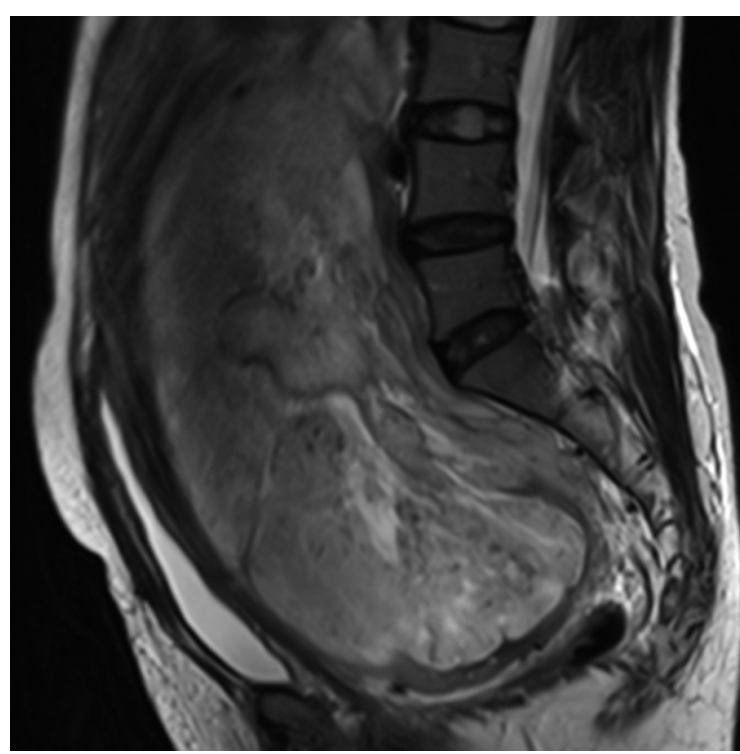

A

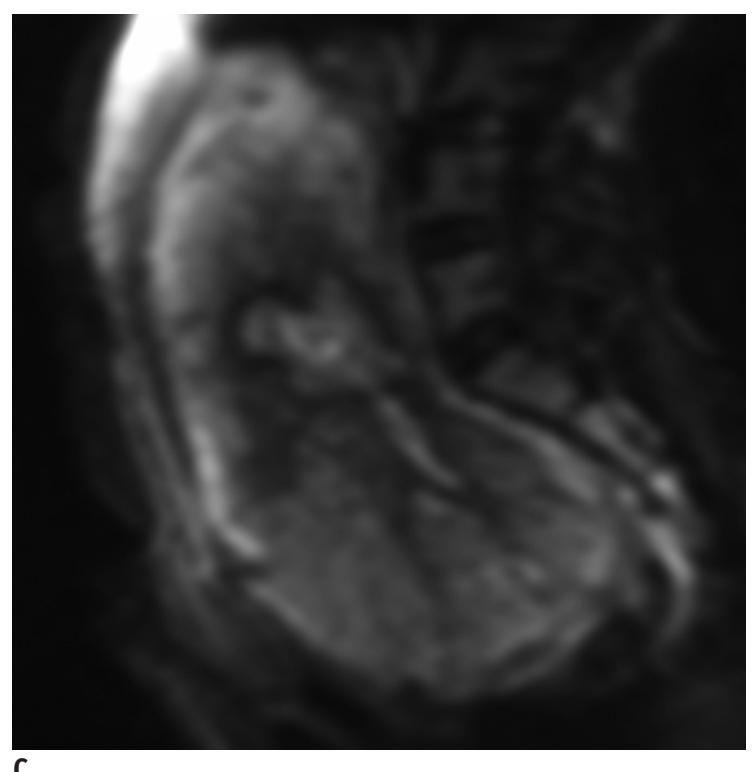

C
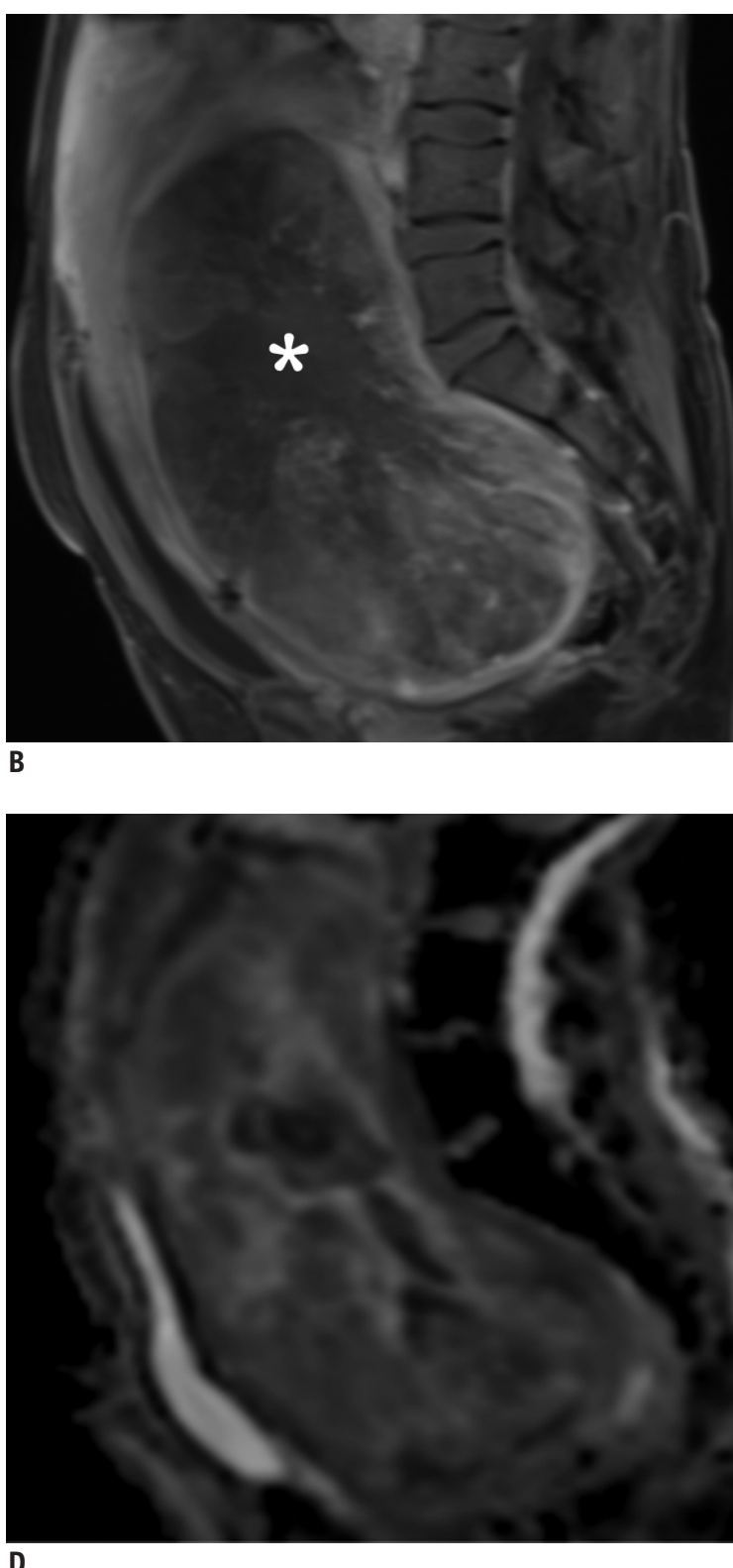

Fig. 1. Characteristic MRI features of LMS. 47-year-old female patient with 19.9-cm LMS at posterior wall of uterus.

A. Sagittal T2WI shows intralesional heterogeneous high signal intensity with distorted inner lining of tumor. B. Post-contrast fat-saturated T1WI shows typical central non-enhancement (asterisk) with hypervascularity of tumor. Diffusion restriction and low signal are observed at periphery of tumor on DWI (C) and ADC map (D) respectively, with ADC value of $1.06 \times 10^{-3} \mathrm{~mm}^{2} / \mathrm{s}$. ADC = apparent diffusion coefficient, DWI = diffusionweighted imaging, $\mathrm{LMS}=$ leiomyosarcoma, MRI = magnetic resonance imaging, $\mathrm{T} 1 \mathrm{WI}=\mathrm{T} 1$-weighted images, $\mathrm{T} 2 \mathrm{WI}=\mathrm{T} 2$-weighted images 
enhanced areas (Fig. 1) are reported to have significantly higher diagnostic accuracy than DWI, T2WI, and T1weighted images (T1WI), in the differentiation between LMS/STUMP and benign leiomyoma (24). CNE correlates with areas of coagulative necrosis, a characteristic feature of LMS on histopathology, due to the lack of interposed granulation or hyalinized tissue to support the LMS tumor architecture resulting in areas of coagulative necrosis deviated by the adjacent viable cells. However, this phenomenon should not be confused with the scattered non-enhancing areas throughout the tumor mass as seen in leiomyomas with hyaline degeneration. False positivity of contrast-enhanced (CE)-MRI is reported in patients with infarcted leiomyomas and requires cautious interpretation $(12,24)$. Hyperintensity on DWI is reported to have high sensitivity but limited specificity in differentiation between LMS/STUMP and benign leiomyoma (19, 20, 25, $26)$. Through the proposed ADC cut-off value of $1.08 \times 10^{-3}$ $\mathrm{mm}^{2} / \mathrm{s}$, the combination of DWI and ADC values can achieve comparable diagnostic accuracy to that of CE-MRI (24). In patients with impaired renal function unsuitable for CE-MRI study, combined DWI and ADC values may offer applicable alterations for diagnosis purpose. Comparative MRI findings in patients with LMS and other uterine sarcomas is summarized in Table 2.

\section{Endometrial Stromal Sarcoma}

\section{ESS: Clinicopathological Features and Diagnostic Challenges \\ Endometrial stromal sarcoma is the second most} common uterine sarcoma $(4,5,27)$. It is composed of cells resembling the endometrial stromal cells at normal proliferative phase. Endometrial stromal and related tumors comprise different subtypes including low-grade ESS, high-grade ESS, and undifferentiated uterine sarcoma (UUS), based on different histopathological features and distinct clinical and prognostic profiles (3). Low-grade ESS tends to occur in patients with younger age than UUS (4), and is characterized by favorable prognosis (28). Staging and vascular invasion are the most important prognostic factors in patients with ESS (27). Patients with either low-grade, high-grade ESS, or UUS confined to the uterus should undergo staging surgery, including total extrafascial hysterectomy with or without bilateral salpingooophorectomy (29). Adjuvant hormone therapy may not improve the prognosis in patients with high-grade ESS or UUS, and therefore, is recommended only in patients with low-grade ESS (5). In general, ESS presents as a sizeable polypoid mass in the endometrial cavity with different degree of myometrial invasion. However, ESS may present as a pure myometrial mass mimicking leiomyoma with degenerative change or atypical features. As in cases with other myometrial-based tumor types, tissue diagnosis using endometrial samplings is not feasible due to the intramuscular location and may result in false negative diagnosis.

\section{ESS: Radiological Advantages and Disadvantages}

Radiologically, ESS is reported as large endometrial-based masses, with well-defined or infiltrative border, sometimes demonstrating myometrial invasion (30). On MRI, ESS presents as a myometrial mass mimicking degenerated leiomyoma or adenomyosis (31), showing characteristic MRI feature of intratumoral T2 hypointense bands scattered

\section{Table 2. Comparison of MRI Findings for Uterine Sarcomas}

\begin{tabular}{|c|c|c|}
\hline Histopathology & Imaging Features & References \\
\hline Leiomyosarcoma & $\begin{array}{l}\text { Large mass with irregular margin, intermediate to high signal intensity on } \\
\text { T2WI, associated with hemorrhage and necrosis, and presence of unenhanced } \\
\text { pocket-like areas especially central non-enhancement on CE-MRI }\end{array}$ & $\begin{array}{l}(10,12,17,19,20, \\
24-26)\end{array}$ \\
\hline Endometrial stromal sarcoma & $\begin{array}{l}\text { Low-grade: endometrial-based heterogeneous mass with or without myometrial } \\
\text { involvement, scattered intratumoral T2 hypointense bands, marginal nodules } \\
\text { and worm-like tumor extension along vessels or ligaments } \\
\text { High-grade: heterogeneous mass with marked vascular and lymphatic invasion, } \\
\text { associated with hemorrhage, necrosis and feather-like enhancement on CE-MRI }\end{array}$ & $(30,31,33-35)$ \\
\hline Adenosarcoma & $\begin{array}{l}\text { Endometrial-based multi-septated cystic mass with low signal intensity solid } \\
\text { areas on T2WI }\end{array}$ & $(4,30,38-40)$ \\
\hline Carcinosarcoma & $\begin{array}{l}\text { Mass that distends endometrial cavity and endocervical canal, exhibiting longer } \\
\text { ratio of anteroposterior diameter to endometrial thickness, with profound and } \\
\text { prolonged enhancement on CE-MRI }\end{array}$ & $(17,47-49,53)$ \\
\hline
\end{tabular}




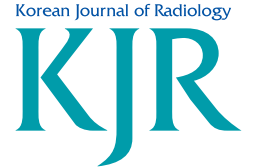

in the areas of myometrial involvement, which represents the preserved myometrial bundles separated by clusters of the tumor cells permeating the myometrium (32). Care should be taken to identify the nodular lesions at the tumor margin, or marginal nodules, which represent the myometrial invasion of tumors (33). Intramyometrial worm-like nodular extensions, defined as detached or discrete nodules from the primary tumor, likewise indicate myometrial and lymphovascular invasion (33). High intensity on DWI with low ADC values are reported in case

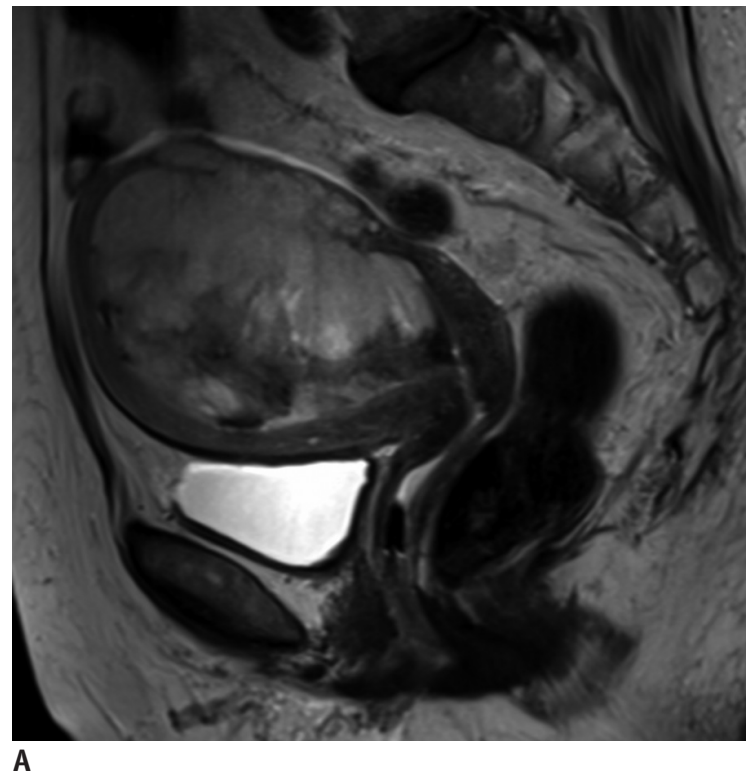

of ESS (20). Compared with EC, ESS tends to show increased and prolonged contrast enhancement (33); and some reports indicate great vessel invasion including the inferior vena cava, heart, or pulmonary vessels (34).

To date, there are few reports describing the different characteristics between low-grade ESS versus high-grade ESS or UUS (30). High-grade ESS is described as the presence of large polypoid masses with heterogeneous appearance and marked vascular and lymphatic invasion. In our experience, feather-like enhancement is the most accurate
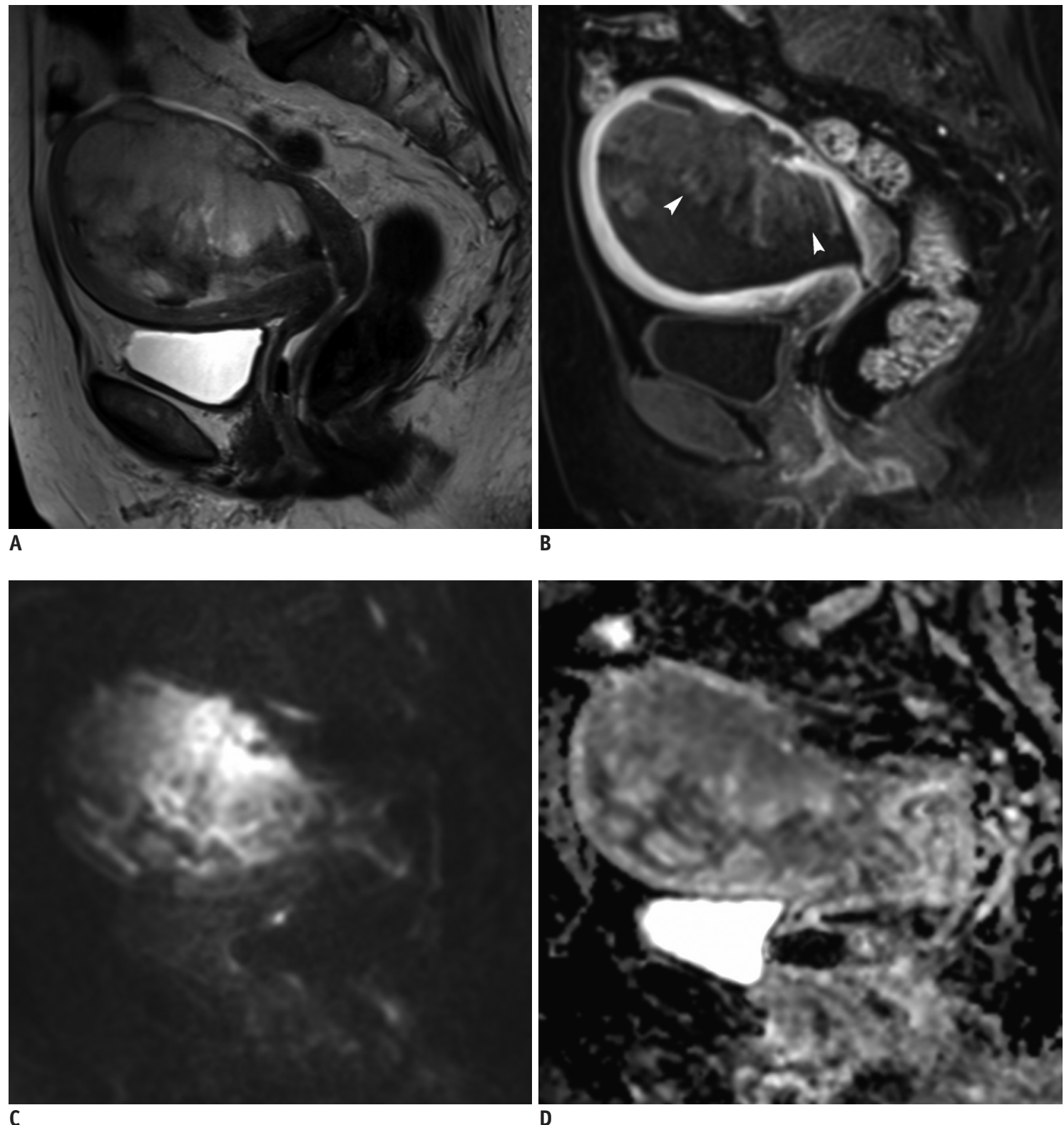

Fig. 2. MRI finding in representative case of high-grade ESS. 88-year-old female patient with 9.0-cm high-grade ESS involving both endometrial cavity and myometrium.

A. Axial T2WI shows tumor signal higher than adjacent myometrium, with scattered intratumoral T2 hypointense bands and marginal nodularity. B. Post-contrast fat-saturated T1WI shows feather-like enhancement (arrowheads). Tumor demonstrates water restriction on DWI (C) and ADC value of $1.01 \times 10^{-3} \mathrm{~mm}^{2} / \mathrm{s}$ on $\mathrm{ADC}$ map (D). ESS = endometrial stromal sarcoma 
MR characteristic of UUS and high-grade ESS (Fig. 2) (35).

\section{Adenosarcoma}

\section{AS: Clinicopathological Features and Diagnostic Challenges}

Uterine adenosarcoma (AS) is extremely rare $(3,4)$. AS is a biphasic tumor comprising benign or atypical epithelial and low-grade malignant mesenchymal components. More than $70 \%$ of AS occurs in the endometrium, but occurrence in the myometrium, cervix, and extra-uterine tissues is reported (36). Most patients with diagnosed AS are postmenopausal women, but premenopausal patients including adolescents may be affected. AS is reported to show association with previous pelvic radiation or longterm unopposed estrogen therapy, mainly tamoxifen (3). The usual presentation includes abnormal vaginal bleeding or large endometrial polypoid-mass lesion. The majority of women with uterine AS present with early-stage tumors and have favorable outcome, with 5 -year survival in $79 \%$ cases with stage I disease (37). Local recurrence of AS occurs in up to $30 \%$ of patients, particularly in the vagina (3). The current staging system incorporates the deep myometrial invasion because of its role as risk factor for recurrence (3). With regard to diagnosis based on pathology of small histological specimens, the sarcomatous stroma of AS can be of very low-grade, which may cause misdiagnosis of benign entity such as adenofibroma or endometrial polyp. AS treated as benign polypoid diseases may show complication of multiple episodes of recurrence $(38,39)$. Therefore, careful consideration of the MRI findings is crucial for selecting proper treatment in patients with AS.

\section{AS: Radiological Advantages and Disadvantages}

Adenosarcoma typically presents as a large welldemarcated polypoid mass located in the endometrial cavity, with tumor mass protruding into the cervical os or vagina in some cases (Fig. 3) (39). The mass usually demonstrates heterogeneous solid components and multi-septated cystic areas with lattice-like appearance due to the intervening septa. The cysts show low signal intensity on T1WI and high signal intensity on T2WI, whereas the solid areas demonstrate small T2 hyperintense foci scattered within the mass, representing glandular epithelial components or necrosis $(4,30,38)$. Areas of high signal intensity on T1WI represent hemorrhage of the tumor. Post-gadolinium images demonstrate enhancement of the solid areas and septa similar to that of the myometrium (30). Studies indicate presence of relatively low water-restriction on DWI, which corresponds to its low-grade $(20,38)$. In women with long-term tamoxifen treatment, AS shares similar imaging features with endometrial polyps, as lattice-like enhancement traversing the endometrial canal (39), with low degree multi-septate cystic areas. Cystic degeneration of the uterine leiomyoma may mimic AS and vice versa (40). AS with MRI feature of the myometrial, peritoneal, or intravascular invasion should be considered as sarcomatous overgrowth (30).

\section{Uterine Carcinosarcoma}

\section{UCS: Clinicopathological Features and Diagnostic Challenges}

Uterine carcinosarcoma, also known as malignant mixed mesodermal tumor or malignant mixed Müllerian tumor (MMMT), is a dedifferentiated carcinoma comprising carcinomatous and sarcomatous elements with highly aggressive behavior. In addition, carcinosarcoma is used for lesions with homologous sarcomatous component, whereas MMMT for lesions with heterologous sarcomatous component. Currently, based on molecular evidence, the metaplastic monoclonal or conversion theory is replacing the combination theory, hence it is recommend to use carcinosarcoma for all lesions with subdivision into homologous and heterologous groups (41). Despite incidence rate of UCS comprising less than $5 \%$ of all uterine malignancies, it leads to mortality rate of more than $16 \%$ (42). UCS typically occurs in postmenopausal women in their $60 \mathrm{~s}$ or $70 \mathrm{~s}$. The most common presenting symptom is postmenopausal bleeding followed by bloody or watery discharge and abdominal pain (43). UCS shares similar risk factors with EC including advanced age, obesity, and exposure to exogenous estrogen, tamoxifen and pelvic radiation (43); and about $60 \%$ of UCS show extrauterine extension at diagnosis with distant metastasis in about $10 \%$ cases, even in patients with apparent earlystage disease (43). Surgery is considered as the primary treatment in patients with UCS, usually combined with ifosfamide/paclitaxel-based adjuvant chemotherapy (5). However, despite all the treatment efforts, the patients' overall survival remains dismal (43). In contrast to other uterine sarcomas, UCS is staged as EC per revised International Federation of Gynecology and Obstetrics (FIGO) 2009 guideline $(5,44)$. MRI shows potential for use in 
preoperative staging in patients with $\mathrm{EC}(45,46)$; however, there is limited available data regarding the accuracy of MRI in the staging of UCS.

\section{UCS: Radiological Advantages and Disadvantages}

Uterine carcinosarcoma most commonly presents as an endometrial mass causing distension of the endometrial cavity and endocervical canal, mimicking myoma delivery (Fig. 4) (47). UCS may be differentiated from EC based on the higher ratio of anteroposterior dimension of the tumor to endometrial thickness (48). UCS typically presents as isointense signal to the myometrium on T1WI and as heterogeneously hyperintense signal on T2WI suggesting intratumoral hemorrhage or necrosis $(47,49)$; and shows prolonged enhancement, in contrast to EC that is usually weakly enhanced $(17,47,49)$. Study shows that similar to the majority of malignant tumors, UCS demonstrates low ADC values on DWI which are significantly higher than
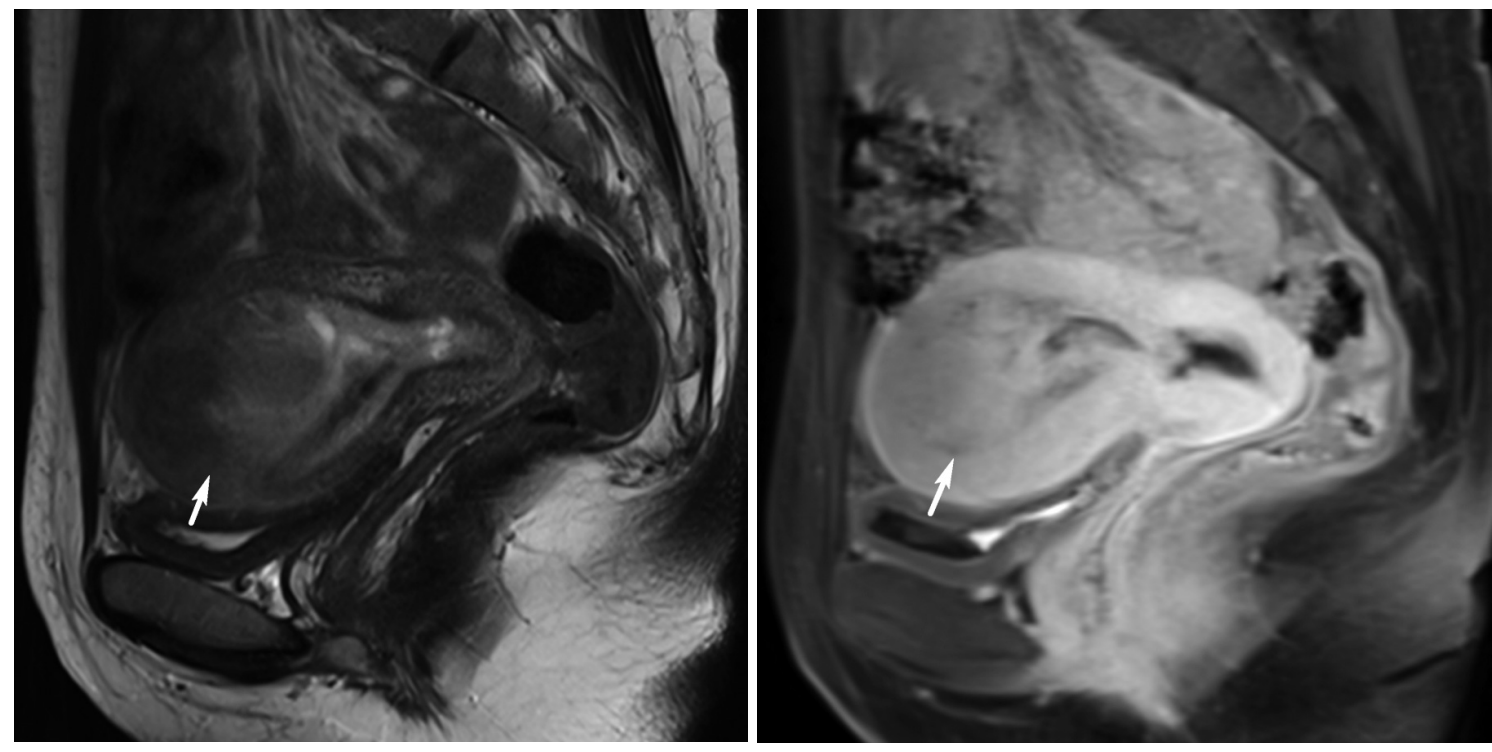

A

B
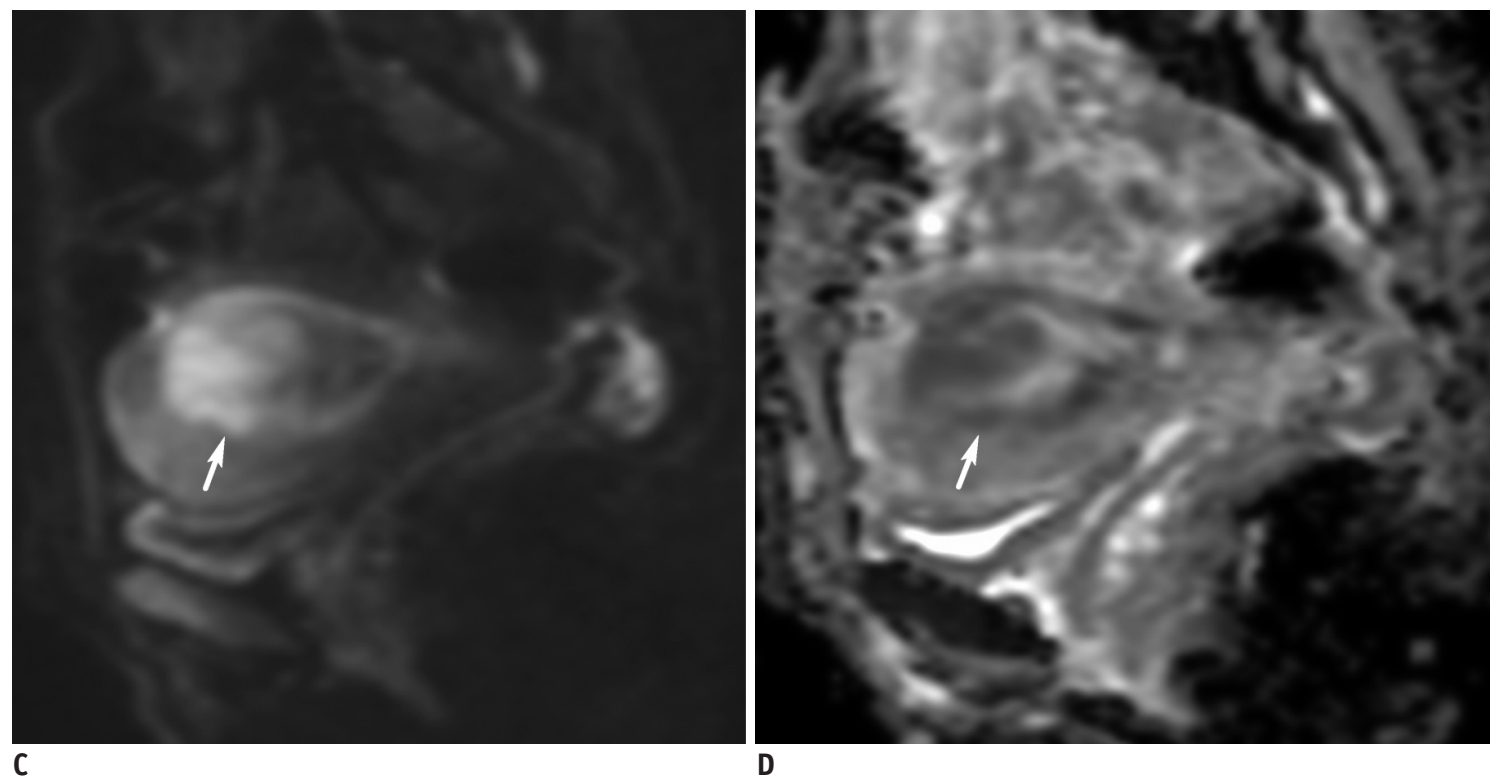

Fig. 3. MRI finding in representative case of AS. 31-year-old woman with 5.6-cm AS located within endometrial cavity (arrows), with superficial myometrial invasion and protrusion into uterine cervix.

A. Axial T2WI shows heterogeneous hyperintensity of tumor with small cyst. B. Post-contrast fat-saturated T1WI shows homogeneous and equal enhancement relative to myometrium with faint lattice-like pattern. Solid component of tumor demonstrates diffusion restriction on DWI (C) and low signal intensity on ADC map (D). ADC value of tumor is $1.19 \times 10^{-3} \mathrm{~mm}^{2} / \mathrm{s}$. AS = adenosarcoma 
that of grade II and III EC (50), corresponding to the hypocellular regions caused by intratumoral necrosis. The collective findings of relatively high mean $A D C$, low choline concentration and high lipid peak on MRS due to intratumoral heterogeneity comprising necrosis and epithelial cystic components may be compatible with the diagnosis of UCS (50). In addition, the ADC map has capability to distinguish between the carcinomatous and sarcomatous components of the UCS (51), based on the presence of greater heterogeneity in the sarcomatous component (52). The presence of cervical stromal invasion and nodal enlargement at presentation are more common in patients with UCS than in those with EC (47). Preoperative DWI is useful to assess the deep myometrial or cervical stromal invasion in patients with uterine UCS; however, its limited diagnostic performance in detecting the adnexal invasion and lymphatic spread requires further improvement (53).

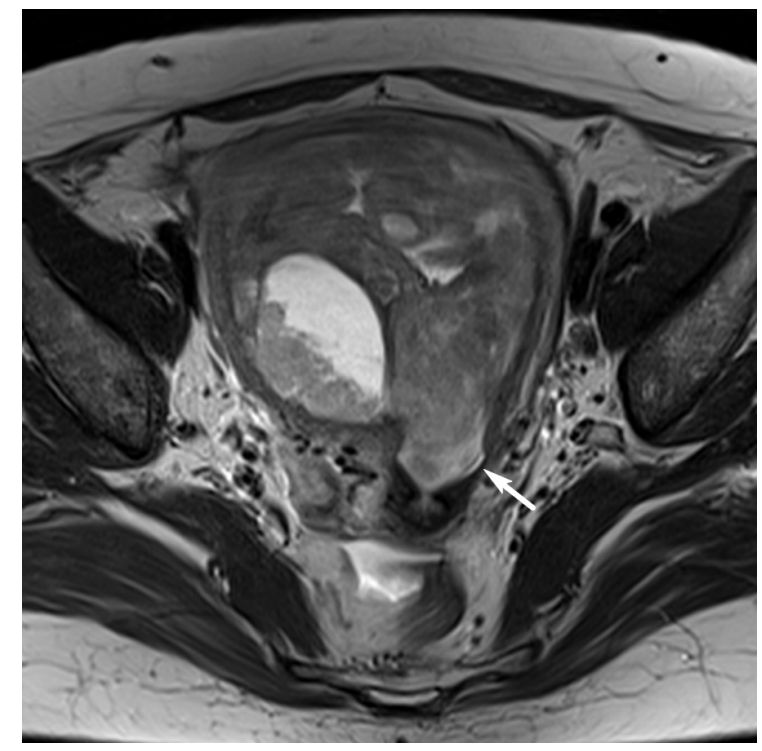

A

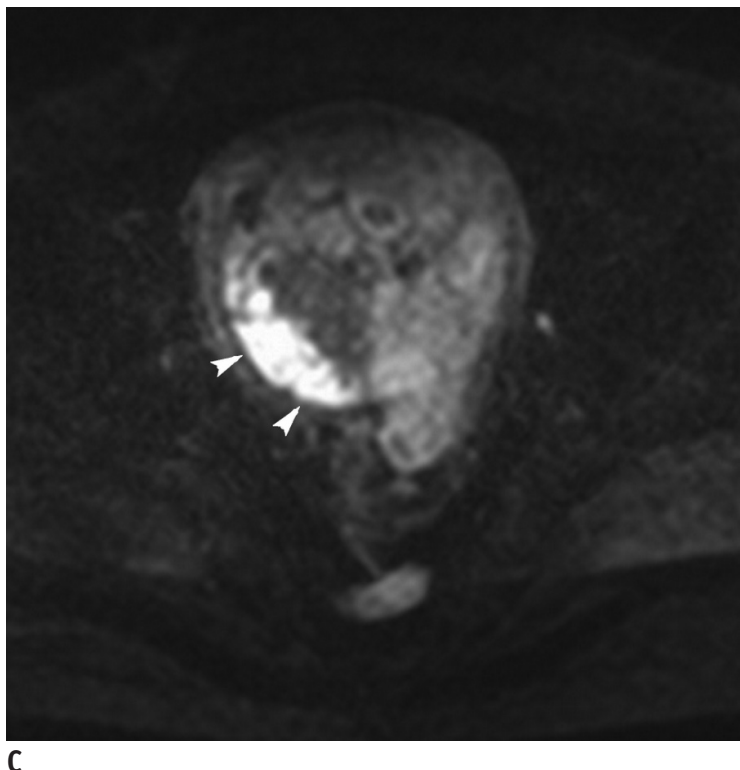

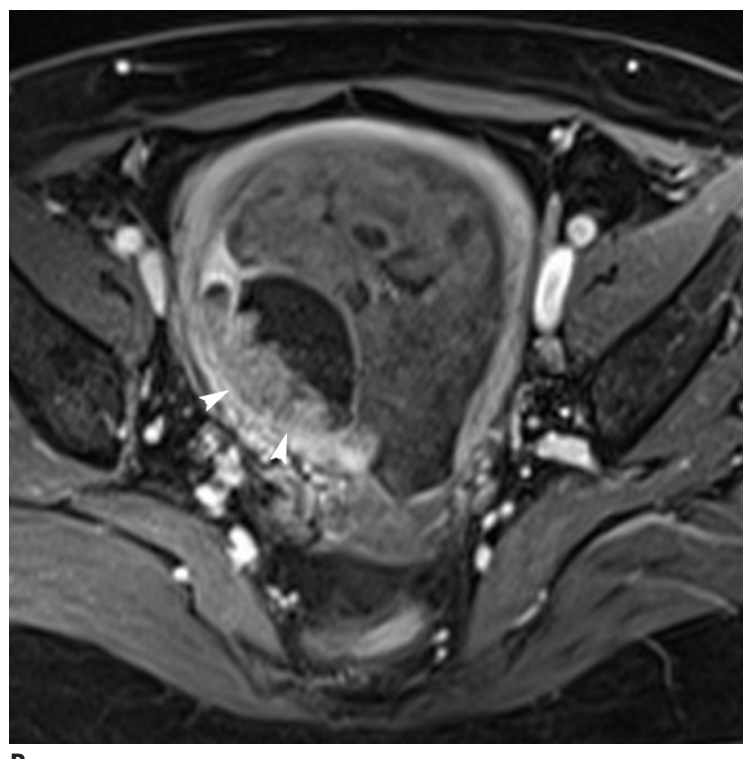

B

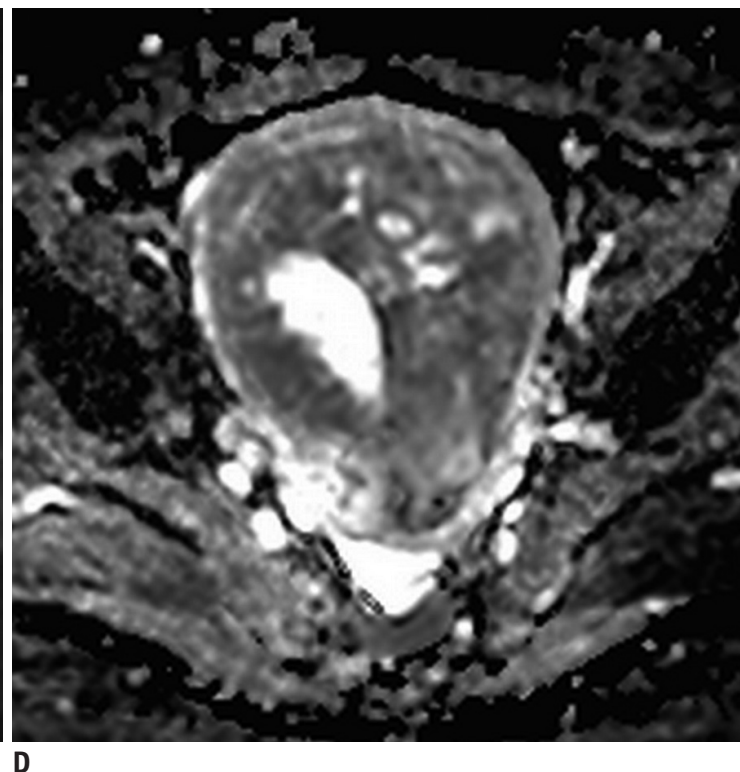

Fig. 4. MRI finding in representative case of uterine carcinosarcoma. 56-year-old woman with 10-cm carcinosarcoma causing distension of uterine cavity.

A. Axial T2WI shows bulky tumor in endometrial cavity with protrusion toward cervical canal, i.e., myoma delivery-like (arrow). Cystic or necrotic portion on right aspect of tumor is observed. B. Post-contrast fat-saturated T1WI and (C) DWI show focal irregular tumor/myometrial interface on right side (arrowheads), corresponding to presence of myometrial invasion based on histopathology. Tumor demonstrates water restriction on DWI and (D) ADC map. ADC value of tumor is $0.97 \times 10^{-3} \mathrm{~mm}^{2} / \mathrm{s}$. 


\section{Endometrial Carcinoma}

\section{EC: Clinicopathological Features and Diagnostic Challenges}

Uterine MRI is mostly indicated in patients with EC, the most common malignancy in the pelvis of female patients in the United States over the past 20 years (1), with increasing incidence in Europe and Asia (2). EC is a relatively manageable disease for gynecological oncologists because patients presenting early symptom of irregular vaginal bleeding seek care at an early and treatable stage of the disease, which is expected to lead to a high survival rate (1). Ultrasonography is a standard tool to evaluate abnormal vaginal bleeding. Based on a $5-\mathrm{mm}$ endometrial thickness threshold, the sensitivity for EC is reported as $96 \%$ and the specificity as $61 \%$ (54). However, in patients with uterine cancer, data shows that the mortality rate increases more rapidly than the incidence rate, which may be related to an increased rate of advanced-stage cancer and high-risk histology type (1). Optimizing the approach to stratify the patients' risks and accordingly develop individualized treatment is an important goal to improve the patient outcome.

Endometrial cancer has two pathogenetic types: type 1 cancers are well or moderately differentiated endometrioid adenocarcinoma with minimal invasion of the myometrium, which are commonly seen in premenopausal women and comprise the majority of EC; whereas, type 2 cancers are poorly differentiated endometrioid, clear cell, or serous papillary type, which may cause deep myometrial invasion, with predominant occurrence in older, post-menopausal populations (55). The recommended histopathologic criteria for determining high-risk disease includes high tumor grade (poorly differentiated), deep myometrial invasion (more than 50\% myometrial thickness), lymphovascular space invasion (LVSI), non-endometrioid histology, and cervical stromal involvement (56).

\section{EC: Myometrial Invasion Depth}

Deep myometrial invasion is the determining factor of the FIGO stage and associated with involvement of the pelvic lymph node (5). The staging performance of MRI in patients with EC is summarized in Table 3. Standard treatment for EC confined to the uterus includes hysterectomy and bilateral salpingo-oophorectomy. Additional surgical pelvic or para-aortic lymphadenectomy is warranted in case of tumor showing deep myometrial invasion, depending on general medical conditions $(5,57)$. Among the imaging tools for evaluation of myometrial invasion, T2-weighted MRI is considered superior to CT and ultrasonography (58). Based on findings through MRI, the presence of intact endometrial/myometrial junction on T2WI and smooth, clear subendometrial enhancement (SEE) on DCE-MRI are indicative of the absence of myometrial invasion; whereas, the irregularity or disruption of SEE and presence of focal strong or irregular peritumoral enhancement (PTE) on DCEMRI are indicative of myometrial invasion. Diagnosis of myometrial invasion based on the disruption of SEE alone may result in increased rate of false positives. Presence of a thin irregular PTE correlates with both the presence and depth of myometrial invasion (59). Over and since the recent decade, DWI is increasingly employed as an assessment tool of the myometrial invasion. The overall diagnostic performance of DWI may be superior to that of DCE-MRI in detecting the deep myometrial invasion, supported by recent reports using a $1.5 \mathrm{~T}$ (45) or 3T MR system (46). Meta-analysis conducted in 2014 shows similar pooled sensitivity under DWI and DCE-MRI; although the specificity is slightly higher under DWI versus DCE-MRI, without significance (60). Further analysis is required to confirm the potential saving in the time and cost of DWI as replacement for additional intravenous contrast medium administration in patients undergoing DCE-MRI, especially elder patients with impaired renal function at risk for nephrogenic systemic fibrosis. Recent technological advancement such as reduced field-of-view DWI enables the correction of distortion and tumor delineation, leading to improved assessment of the deep myometrial invasion (61-63). Although not included in the updated 2009 FIGO staging system, clinical exclusion of myometrial involvement is critical in nulliparous patients younger than 40 years, because fertility-preserving treatment may be an option for such patients with pathologically proven type 1 cancer and normal serum levels of cancer antigen 125 (CA 125) (64). Uterine morphology should be carefully interpreted under condition of changes based on the patients' menopausal status. The myometrial-endometrial interfaces on T2WI may be obscure in postmenopausal women due to hormonerelated atrophy and fibrous involution of the uterine smooth muscle tissue. In premenopausal patients with EC planning to undergo fertility-preserving treatment, DCE-MRI is superior to DWI in terms of excluding the possibility of the myometrial invasion (65). 
Table 3. Diagnostic Performance of MRI for Endometrial Cancer

\begin{tabular}{|c|c|c|c|c|}
\hline & Sensitivity (\%) & Specificity (\%) & Accuracy $(\%)$ & References \\
\hline \multicolumn{5}{|l|}{ Any myometrial invasion } \\
\hline Mixed age group & & & & $(46)$ \\
\hline T2WI & 88 & 73 & 83 & \\
\hline DWI & 94 & 80 & 90 & \\
\hline DCE-MRI & 85 & 60 & 77 & \\
\hline Premenopausal & & & & $(65)$ \\
\hline DWI & $25-75$ & $37-63$ & $42-58$ & \\
\hline DCE-MRI & 100 & $48-56$ & $55-61$ & \\
\hline Postmenopausal & & & & (65) \\
\hline DWI & $77-81$ & $53-65$ & 73 & \\
\hline DCE-MRI & $79-84$ & $47-53$ & $72-73$ & \\
\hline \multicolumn{5}{|l|}{ Deep myometrial invasion } \\
\hline Mixed age group & & & & $(45,46,60)$ \\
\hline T2WI & 57 & 81 & 77 & \\
\hline DWI & $85-90$ & $84-100$ & $88-100$ & \\
\hline DCE-MRI & $71-100$ & $61-93$ & $82-94$ & \\
\hline Premenopausal & & & & (65) \\
\hline DWI & $\mathrm{N} / \mathrm{A}$ & $94-100$ & $94-100$ & \\
\hline DCE-MRI & $\mathrm{N} / \mathrm{A}$ & $97-100$ & $97-100$ & \\
\hline Postmenopausal & & & & $(65)$ \\
\hline DWI & 90 & $95-98$ & $93-95$ & \\
\hline DCE-MRI & $70-85$ & $88-93$ & $85-87$ & \\
\hline Cervical stroma invasion & & & & $(67,68,70)$ \\
\hline Overall performance & $50-100$ & $78-97$ & $69-95$ & \\
\hline T2WI & $50-67$ & $78-86$ & $76-81$ & \\
\hline DWI & $92-100$ & $90-96$ & $92-95$ & \\
\hline DCE-MRI & $50-58$ & $94-97$ & $88-92$ & \\
\hline Pelvic nodal metastases & $50-85$ & $90-99$ & $77-99$ & $(84,86,87)$ \\
\hline
\end{tabular}

$\mathrm{DCE}=$ dynamic contrast enhancement

\section{EC: Cervical Stromal Invasion}

The presence of cervical stromal invasion represents FIGO stage II disease in patients with EC (57), which is directly associated with higher risk of spread to the lymph nodes and a poor prognostic factor (56). Moreover, it is an indication for radical versus simple hysterectomy (5), and administration of adjuvant brachytherapy (66). MRI is more accurate than endocervical curettage and hysteroscopy in the prediction of stromal invasion (67). According to recent meta-analysis, the pooled specificity of conventional MRI in the prediction of cervical stromal invasion is $95 \%$, whereas the sensitivity is only $57 \%(68)$. In general, stromal invasion of the cervix is most clearly depicted on the sagittal and axial oblique T2WI as an area of hypointense cervical stroma disrupted by tumors. DCE-MRI is considered as state-of-the-art standard for tumor delineation (68). However, diagnosis is difficult in case of the presence of endometrial tumors within the endocervical canal, with widening of the internal os, and obliterating of the interface between the tumor and cervical stroma (69). Recent study indicates further improvement in the performance of DWI in detecting the cervical stromal invasion (Fig. 5) (70).

\section{EC: Tumor ADC Value}

The ADC values are associated with the tumor grades in patients with EC $(65,71)$. Nougaret et al. (72) report significantly lower volumetric $A D C$ values in patients with grade III EC and tumors with lymphovascular invasion. Quartile ADC values are higher (73) but minimum tumor $A D C$ values are lower in tumors showing invasion of the cervical stroma (74). A recent study shows that the cholineto-water ratio increases with the increasing tumor stage and large tumor size in patients with EC (75). Furthermore, the choline-to-noise ratio is reported as significantly higher in type 2 ECs than that in type 1 EC (76). Under condition of combined morphology and MRS test, the tumor 

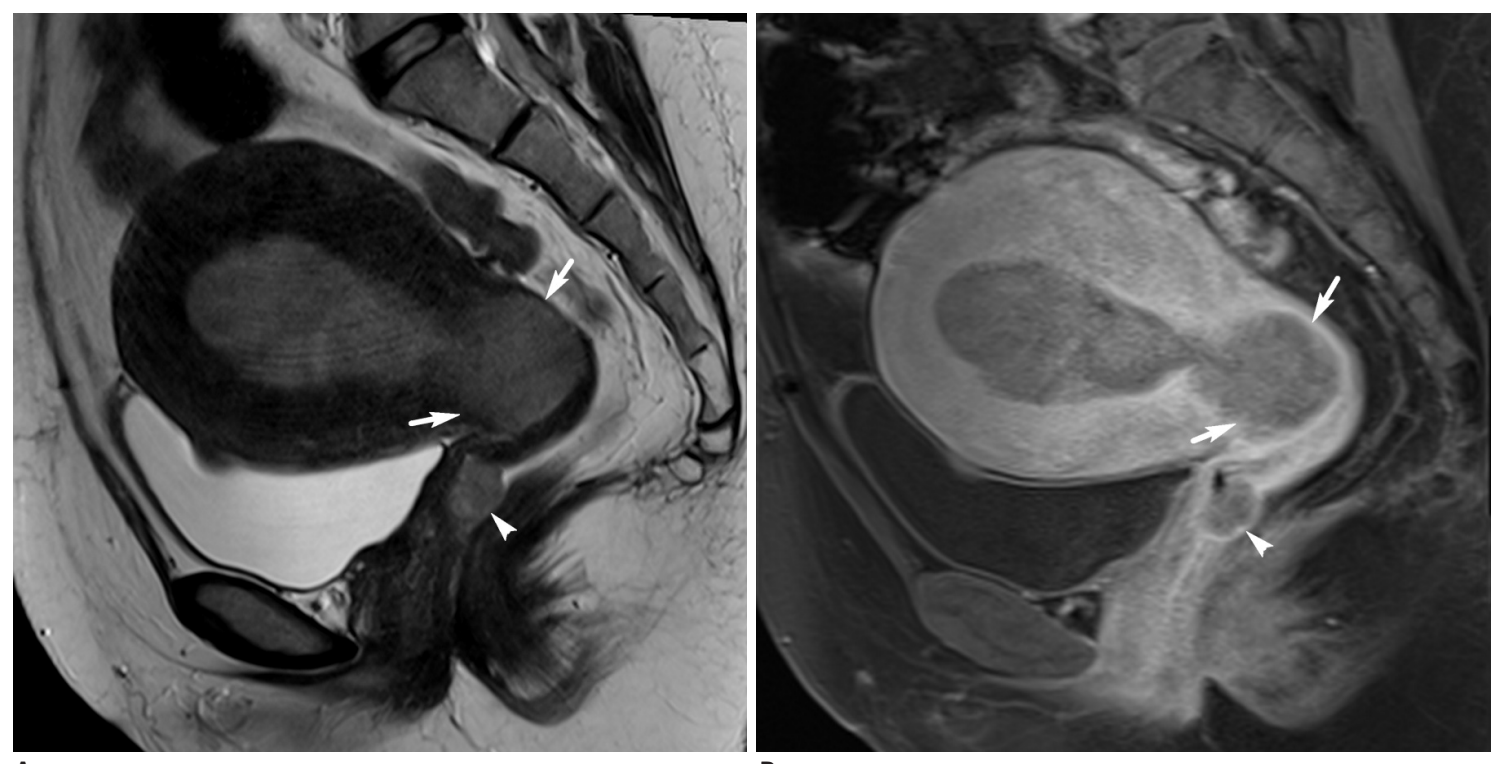

A

B
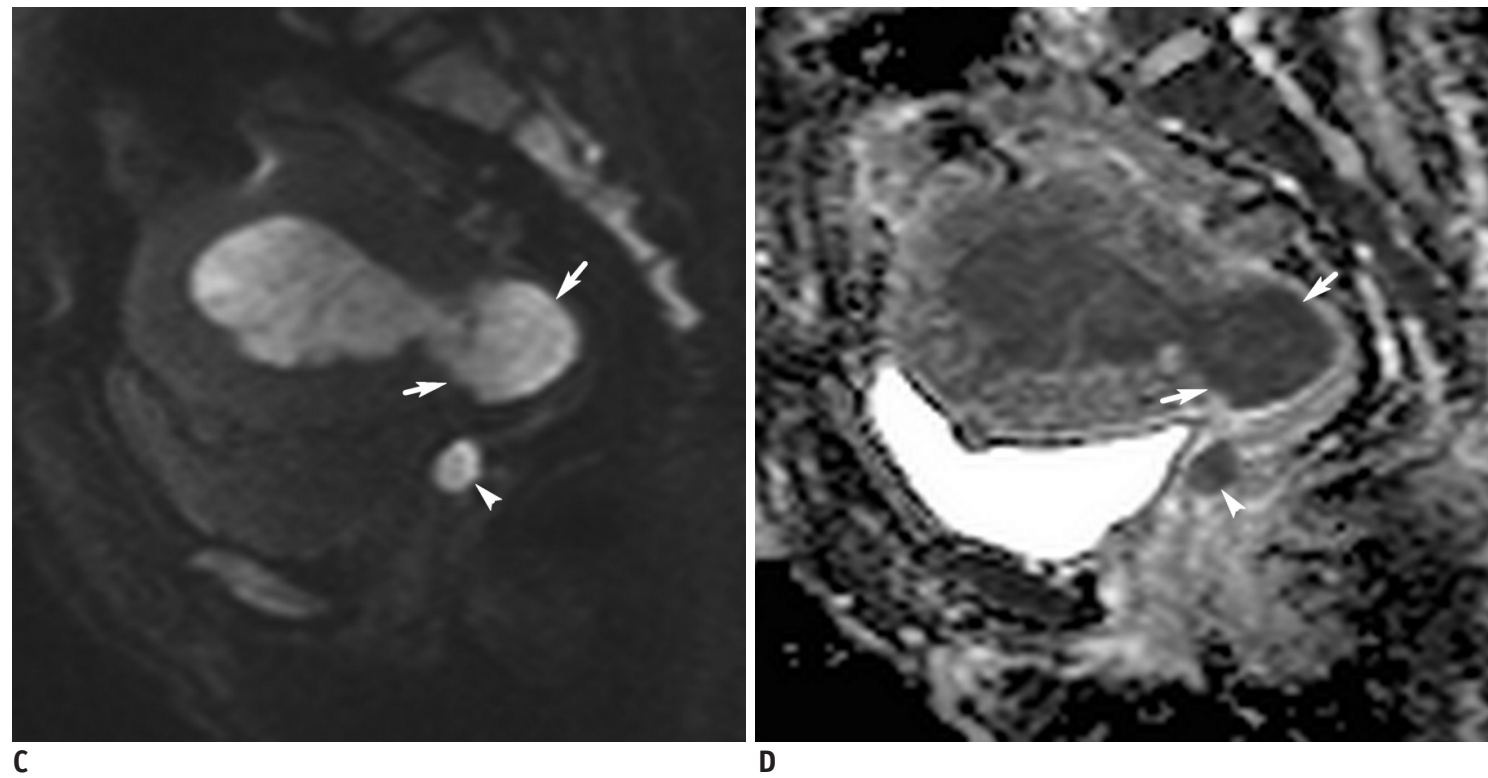

Fig. 5. MRI finding in representative case of EC with cervical stromal invasion. 44-year-old woman with 10.6- $\mathrm{cm}$ tumor with cervical stromal invasion.

(A) Sagittal T2WI (B) post-contrast fat-saturated T1WI (C) DWI in $b=1000 \mathrm{~s} / \mathrm{mm}^{2}$ (D) ADC map shows tumor with myometrial invasion and cervical stromal invasion (arrows). Satellite lesion at upper vaginal wall (arrowheads) with similar signal intensity as that of primary tumor is confirmed as metastasis. ADC value of tumor is $0.69 \times 10^{-3} \mathrm{~mm}^{2} / \mathrm{s}$. Based on histopathology, diagnosis was poorly differentiated endometrioid carcinoma. $\mathrm{EC}=$ endometrial cancer

$A D C$ values provide quantitative estimate to differentiate between the $\mathrm{EC}$ with invasion of the cervical stroma and the adenocarcinoma originating from the cervix (11). In contrast, the ADC map is useful for tumor delineation through visual inspection to distinguish the diffusionrestricted lesions from those with the T2 shine-through effect caused by benign fluid-containing structures, such as inflamed tissue and nabothian cysts of the cervix (8). Artificial intelligence (AI) and machine learning refer to computer algorithms that show improved performance with increasing data input, and deep learning is the subtype that attracts particular interest in the field of radiology; however, the evidence related to use of these approaches in patients with uterine malignancy is limited. Recent report describes a machine learning approach using random forest prediction model based on 180 MRI-based texture features combining T2WI, DCE-MRI, and ADC map (77), indicating diagnostic accuracy in case of the deep myometrial invasion 
of $81 \%$, LVSI of $77 \%$, and high-grade tumor of $78 \%$.

However, the preliminary results should be interpreted with caution; further study including an independent dataset is required to validate these results, because the small sample size relative to the number of features extracted may have resulted in over-fitting of the models.

\section{EC: Lymph Node Metastasis}

The prognosis in patients with $\mathrm{EC}$ with nodal involvement is poor, with a 5-year overall survival rate of $51 \%$ (78). Since the presence of nodal metastasis significantly affects the patient's prognosis and provides indication of suitable therapeutic option, surgical staging including systematic lymphadenectomy is recommended as part of comprehensive case-management practice in such patients (79). The recent debate focused on the value of routine lymphadenectomy in patients with EC comprises a three-fold controversy: first, randomized trials suggest the absence of survival benefit

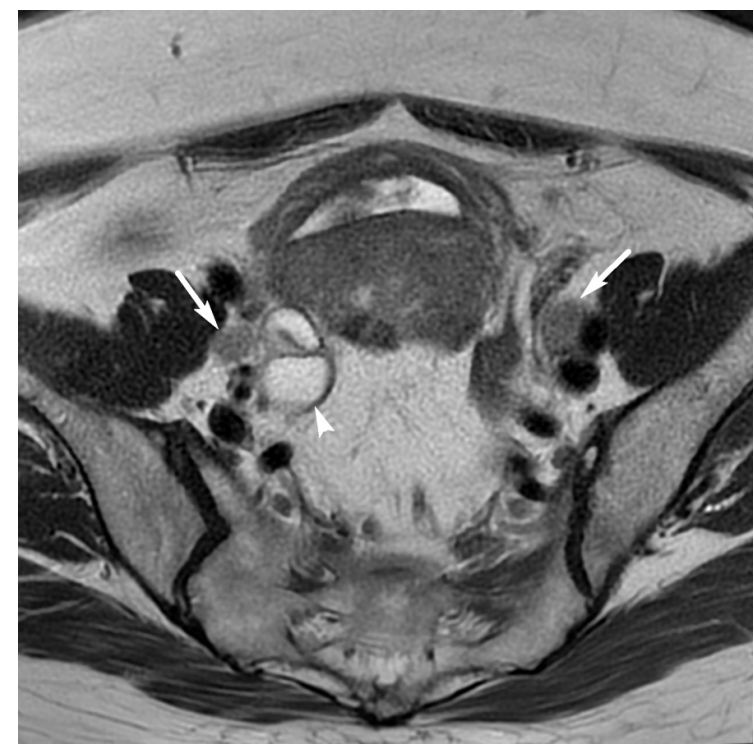

A

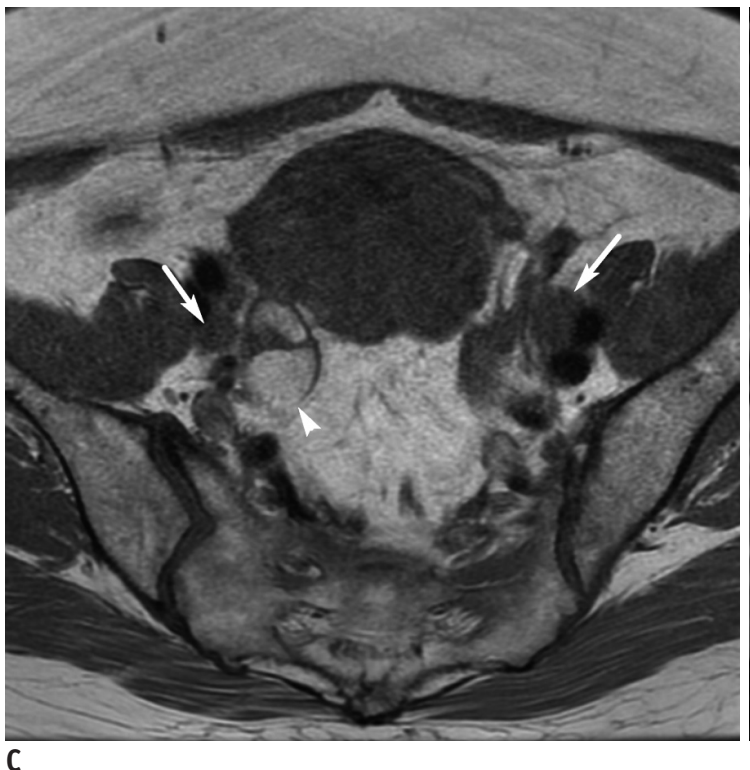

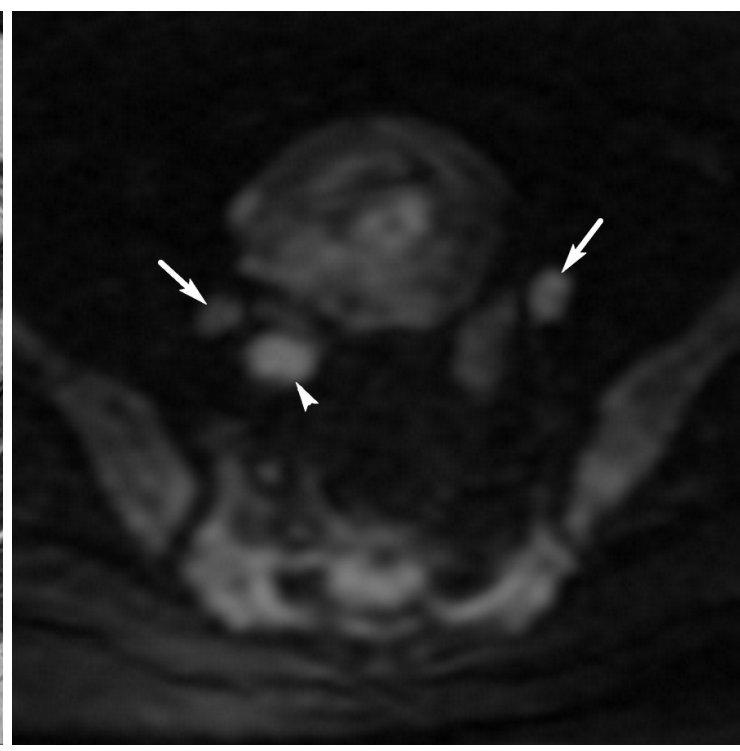

B

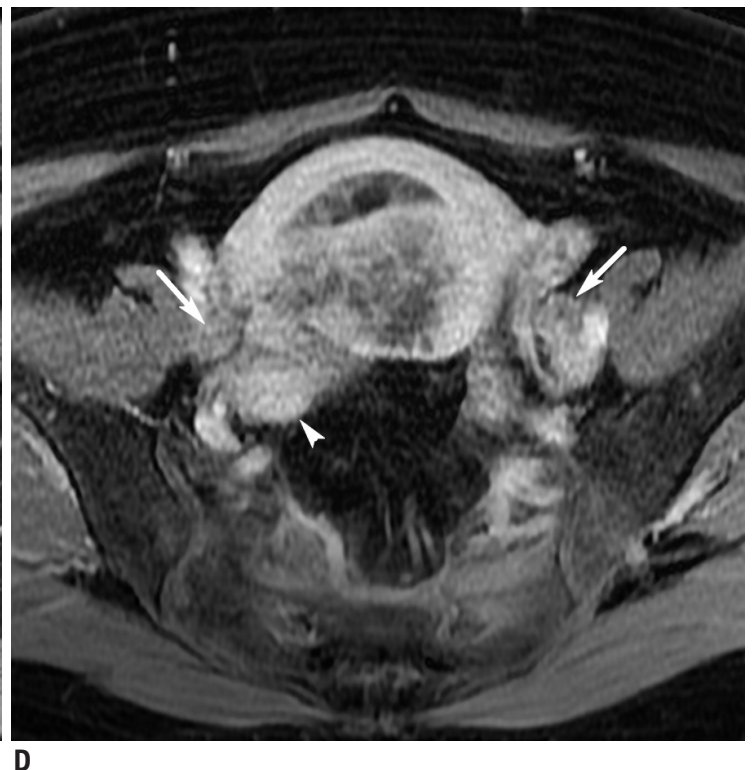

Fig. 6. MRI finding in representative case of EC with pelvic lymph node metastasis. 64-year-old woman with 9.5-cm endometrioid carcinoma with $93 \%$ myometrial invasion.

(A) Axial T2WI, (B) DWI in b $=1000 \mathrm{~s} / \mathrm{mm}^{2}$, (C) T1WI and (D) contrast-enhanced fat-saturated T1WI show enlarged bilateral external iliac lymph nodes (arrows) with diffusion-restriction. Right hydrosalpinx (arrowheads) with focal high signal intensity on DWI and hyperintensity on T1WI, representing hemorrhage. Bilateral pelvic lymph node metastasis and right ovarian metastasis was confirmed based on final pathology result. $A D C$ value of tumor is $0.71 \AA \sim 10^{-3} \mathrm{~mm}^{2} / \mathrm{s}$. 
in patients undergoing systematic lymphadenectomy; second, potential post-operative morbidity following lymphadenectomy may jeopardize the patients' quality of life; and third, difficulty in procedural lymphadenectomy in obese patients is gradually increasing in a substantial proportion of the patient population $(80,81)$. Emerging evidence supports the survival benefit of systematic lymphadenectomy in select patients with $\mathrm{EC}$ at intermediate or high risk for nodal metastasis $(82,83)$. This highlights the importance of risk stratification in patients with indication of lymphadenectomy, to balance the therapeutic benefit against perioperative morbidity and mortality. However, in current clinical setting, optimal pre-operative risk assessment remains difficult (83).

With regard to evaluating the lymph node metastasis, threshold diameter of $10 \mathrm{~mm}$ in the short axis is commonly applied in the conventional MRI protocol, with sensitivity range, $24-73 \%$ (84). The criterion of combined size with relative $A D C$ values on DWI shows potential in detecting the pelvic lymph node metastasis in patients with EC, providing better sensitivity and maintaining similar specificity, as compared with conventional MR (Fig. 6). Using this method, the threshold value in terms of detectable size of the lymph node with metastasis is short-axis of $5 \mathrm{~mm}$ (84). This issue is particularly important in patients with UCS at high risk of nodal metastasis than patients with EC (47). Park et al. (85) report that PET-CT has better sensitivity and specificity than MRI for both pelvic and para-aortic lymph node metastases in patients with EC. With regard to detecting the pelvic lymph node in patients with EC, PET-CT shows better performance than MRI in terms of sensitivity (67$74 \%$ vs. $79 \%$ ); whereas, specificity is similar between the two techniques (93\%) (86). With regard to detecting the para-aortic lymph node, PET-CT shows better performance than MRI in terms of sensitivity ( $83 \%$ vs. $67 \%$ ); whereas, specificity is similar between the two techniques $(100 \%)$ (86). With combined DCE and DWI, MRI shows limited diagnostic accuracy in detecting metastasis to the pelvic lymph node, but high specificity in detecting metastasis to the pelvic lymph node (87). The retrospective fused PET and MRI-DWI (PET/MRI-DWI) shows equal performance to PET$\mathrm{CT}$, with sensitivity of $88 \%$ and specificity of $84 \%$ on perpatient basis; and PET/MRI-DWI show better performance than PET-CT, with sensitivity ( $89 \%$ vs. $70 \%$ ) and specificity (92\% vs. $91 \%$ ) on per-lesion basis (88).

\section{CONCLUSION}

In summary, MRI and MRS play essential roles in the casemanagement of malignant uterine neoplasms, including tumor characterization, primary staging, and treatment planning. MRI shows characteristic features that may be used to differentiate the uterine sarcomas. Combined with DWI and DCE techniques, the advancements in MRI enable more precise assessment of the deep myometrial or cervical stromal invasion in patients with uterine malignancies; however, further investigation is needed to improve its limited diagnostic performance in patients with the adnexal invasion and lymphatic spread. Despite limited evidencebased applicability of AI and machine learning in patients with uterine malignancy, radiologists who are aware of the principles and potential applications of machine learning may have the opportunity to facilitate its translation to clinical use. Emerging technologies combined with AI in MRI have potential to progressively improve the casemanagement of uterine malignancies and improve the patients' outcomes.

\section{Conflicts of Interest}

The authors have no financial conflicts of interest.

\section{Acknowledgments}

We would like to express sincere gratitude to patients and their families who inspire and motivate us. We are indebted to our colleagues in Departments of Pathology, Nuclear Medicine, Gynecology Oncology and Radiation Oncology, for their indepth discussions on weekly multidisciplinary tumor board.

\section{ORCID}

Gigin Lin

https://orcid.org/0000-0001-7246-1058

Yu-Ting Huang

https://orcid.org/0000-0002-7451-0690

Yen-Ling Huang

https://orcid.org/0000-0003-2699-8350

\section{REFERENCES}

1. Siegel RL, Miller KD, Jemal A. Cancer statistics, 2018. CA Cancer J Clin 2018;68:7-30

2. Global Cancer Observatory. International Agency for Research on Cancer (IARC) web site. http://globocan.iarc.fr/. Published 2014. Accessed May 11, 2018

3. Kurman RJ, Carcangiu ML, Herrington CS, Young RH. WHO 
classification of tumours of female reproductive organs, Volume 6, 4th ed. Geneva: World Health Organization, 2014:141-145

4. D’Angelo E, Prat J. Uterine sarcomas: a review. Gynecol Oncol 2010;116:131-139

5. Koh WJ, Abu-Rustum NR, Bean S, Bradley K, Campos SM, Cho KR, et al. Uterine neoplasms, Version 1.2018, NCCN clinical practice guidelines in oncology. J Natl Compr Canc Netw 2018;16:170-199

6. Patel-Lippmann K, Robbins JB, Barroilhet L, Anderson B, Sadowski EA, Boyum J. MR imaging of cervical cancer. Magn Reson Imaging Clin N Am 2017;25:635-649

7. Sala E, Rockall AG, Freeman SJ, Mitchell DG, Reinhold C. The added role of MR imaging in treatment stratification of patients with gynecologic malignancies: what the radiologist needs to know. Radiology 2013;266:717-740

8. Padhani AR, Koh DM, Collins DJ. Whole-body diffusionweighted MR imaging in cancer: current status and research directions. Radiology 2011;261:700-718

9. Leach M0, Morgan B, Tofts PS, Buckley DL, Huang W, Horsfield $M A$, et al.; Experimental Cancer Medicine Centres Imaging Network Steering Committee. Imaging vascular function for early stage clinical trials using dynamic contrast-enhanced magnetic resonance imaging. Eur Radiol 2012;22:1451-1464

10. Goto A, Takeuchi S, Sugimura K, Maruo T. Usefulness of Gd-DTPA contrast-enhanced dynamic MRI and serum determination of LDH and its isozymes in the differential diagnosis of leiomyosarcoma from degenerated leiomyoma of the uterus. Int J Gynecol Cancer 2002;12:354-361

11. Lin G, Lin YC, Wu RC, Yang LY, Lu HY, Tsai SY, et al. Developing and validating a multivariable prediction model to improve the diagnostic accuracy in determination of cervical versus endometrial origin of uterine adenocarcinomas: a prospective MR study combining diffusion-weighted imaging and spectroscopy. J Magn Reson Imaging 2018;47:1654-1666

12. Cornfeld D, Israel G, Martel M, Weinreb J, Schwartz P, McCarthy S. MRI appearance of mesenchymal tumors of the uterus. Eur J Radiol 2010;74:241-249

13. Parker WH, Fu YS, Berek JS. Uterine sarcoma in patients operated on for presumed leiomyoma and rapidly growing leiomyoma. Obstet Gynecol 1994;83:414-418

14. Giuntoli RL 2nd, Metzinger DS, DiMarco CS, Cha SS, Sloan JA, Keeney GL, et al. Retrospective review of 208 patients with leiomyosarcoma of the uterus: prognostic indicators, surgical management, and adjuvant therapy. Gynecol Oncol 2003;89:460-469

15. Wu TI, Yen TC, Lai CH. Clinical presentation and diagnosis of uterine sarcoma, including imaging. Best Pract Res Clin Obstet Gynaecol 2011;25:681-689

16. Lakhman Y, Veeraraghavan H, Chaim J, Feier D, Goldman DA, Moskowitz CS, et al. Differentiation of uterine leiomyosarcoma from atypical leiomyoma: diagnostic accuracy of qualitative MR imaging features and feasibility of texture analysis. Eur Radiol 2017;27:2903-2915

17. Shah SH, Jagannathan JP, Krajewski K, O’Regan KN, George
S, Ramaiya NH. Uterine sarcomas: then and now. AJR Am J Roentgenol 2012;199:213-223

18. Tanaka Y0, Nishida M, Tsunoda H, Okamoto Y, Yoshikawa H. Smooth muscle tumors of uncertain malignant potential and leiomyosarcomas of the uterus: MR findings. J Magn Reson Imaging 2004;20:998-1007

19. Thomassin-Naggara I, Dechoux S, Bonneau C, Morel A, Rouzier R, Carette MF, et al. How to differentiate benign from malignant myometrial tumours using MR imaging. Eur Radiol 2013;23:2306-2314

20. Tamai K, Koyama T, Saga T, Morisawa N, Fujimoto K, Mikami $Y$, et al. The utility of diffusion-weighted MR imaging for differentiating uterine sarcomas from benign leiomyomas. Eur Radiol 2008;18:723-730

21. Namimoto T, Yamashita Y, Awai K, Nakaura T, Yanaga Y, Hirai $T$, et al. Combined use of T2-weighted and diffusion-weighted 3-T MR imaging for differentiating uterine sarcomas from benign leiomyomas. Eur Radiol 2009;19:2756-2764

22. Ueda H, Togashi K, Konishi I, Kataoka ML, Koyama T, Fujiwara $T$, et al. Unusual appearances of uterine leiomyomas: MR imaging findings and their histopathologic backgrounds. Radiographics 1999;19 Spec No:S131-S145

23. Hricak H, Tscholakoff D, Heinrichs L, Fisher MR, Dooms $\mathrm{GC}$, Reinhold C, et al. Uterine leiomyomas: correlation of MR, histopathologic findings, and symptoms. Radiology 1986;158:385-391

24. Lin G, Yang LY, Huang YT, Ng KK, Ng SH, Ueng SH, et al. Comparison of the diagnostic accuracy of contrast-enhanced MRI and diffusion-weighted MRI in the differentiation between uterine leiomyosarcoma / smooth muscle tumor with uncertain malignant potential and benign leiomyoma. J Magn Reson Imaging 2016;43:333-342

25. Li HM, Liu J, Qiang JW, Zhang H, Zhang GF, Ma F. Diffusionweighted imaging for differentiating uterine leiomyosarcoma from degenerated leiomyoma. J Comput Assist Tomogr 2017;41:599-606

26. Sato K, Yuasa N, Fujita M, Fukushima Y. Clinical application of diffusion-weighted imaging for preoperative differentiation between uterine leiomyoma and leiomyosarcoma. Am J Obstet Gynecol 2014;210:368.e1-e368.e8

27. Tropé CG, Abeler VM, Kristensen GB. Diagnosis and treatment of sarcoma of the uterus. A review. Acta Oncol 2012;51:694705

28. Tse KY, Crawford R, Ngan HY. Staging of uterine sarcomas. Best Pract Res Clin Obstet Gynaecol 2011;25:733-749

29. Gadducci A, Cosio S, Romanini A, Genazzani AR. The management of patients with uterine sarcoma: a debated clinical challenge. Crit Rev Oncol Hematol 2008;65:129-142

30. Tirumani SH, Ojili V, Shanbhogue AK, Fasih N, Ryan JG, Reinhold C. Current concepts in the imaging of uterine sarcoma. Abdom Imaging 2013;38:397-411

31. Furukawa R, Akahane M, Yamada H, Kiryu S, Sato J, Komatsu S, et al. Endometrial stromal sarcoma located in the myometrium with a low-intensity rim on T2-weighted images: report of 
three cases and literature review. J Magn Reson Imaging 2010;31:975-979

32. Koyama T, Togashi K, Konishi I, Kobayashi H, Ueda H, Kataoka $M L$, et al. MR imaging of endometrial stromal sarcoma: correlation with pathologic findings. AJR Am J Roentgenol 1999;173:767-772

33. Ueda M, Otsuka M, Hatakenaka M, Sakai S, Ono M, Yoshimitsu $K$, et al. MR imaging findings of uterine endometrial stromal sarcoma: differentiation from endometrial carcinoma. Eur Radiol 2001;11:28-33

34. Boskovic V, Bozanovic T, Ljubic A, Likic-Ladjevic I, Janjic T, Milicevic S. Endometrial stromal sarcoma with intracaval extension at initial presentation. Eur J Gynaecol Oncol 2013;34:280-281

35. Huang YL, Huang YT, Ng KK, Lai CH, Lin G. Differentiating low-grade endometrial stromal sarcoma and undifferentiated endometrial sarcoma by using magnetic resonance imaging, Abstract No. ESG07-0441. Proceeding of International Meeting of the European Society of Gynaecological Oncology;2017 November 4-7; Vienna, Austria

36. Santos P, Cunha TM. Uterine sarcomas: clinical presentation and MRI features. Diagn Interv Radiol 2015;21:4-9

37. Arend R, Bagaria M, Lewin SN, Sun X, Deutsch I, Burke WM, et al. Long-term outcome and natural history of uterine adenosarcomas. Gynecol Oncol 2010;119:305-308

38. Takeuchi M, Matsuzaki K, Yoshida S, Kudo E, Bando Y, Hasebe $\mathrm{H}$, et al. Adenosarcoma of the uterus: magnetic resonance imaging characteristics. Clin Imaging 2009;33:244-247

39. Yoshizako T, Wada A, Kitagaki H, Ishikawa N, Miyazaki K. MR imaging of uterine adenosarcoma: case report and literature review. Magn Reson Med Sci 2011;10:251-254

40. Barral M, Placé V, Dautry R, Bendavid S, Cornelis F, Foucher R, et al. Magnetic resonance imaging features of uterine sarcoma and mimickers. Abdom Radiol (NY) 2017;42:1762-1772

41. Artioli G, Wabersich J, Ludwig K, Gardiman MP, Borgato L, Garbin F. Rare uterine cancer: carcinosarcomas. Review from histology to treatment. Crit Rev Oncol Hematol 2015;94:98104

42. Arend R, Doneza JA, Wright JD. Uterine carcinosarcoma. Curr Opin Oncol 2011;23:531-536

43. Menczer J. Review of recommended treatment of uterine carcinosarcoma. Curr Treat Options Oncol 2015;16:53

44. Lewin SN. Revised FIG0 staging system for endometrial cancer. Clin Obstet Gynecol 2011;54:215-218

45. Beddy P, Moyle P, Kataoka M, Yamamoto AK, Joubert I, Lomas $D$, et al. Evaluation of depth of myometrial invasion and overall staging in endometrial cancer: comparison of diffusion-weighted and dynamic contrast-enhanced MR imaging. Radiology 2012;262:530-537

46. Lin G, Ng KK, Chang CJ, Wang JJ, Ho KC, Yen TC, et al. Myometrial invasion in endometrial cancer: diagnostic accuracy of diffusion-weighted 3.0-T MR imaging--initial experience. Radiology 2009;250:784-792

47. Bharwani N, Newland A, Tunariu N, Babar S, Sahdev A, Rockall
$A G$, et al. MRI appearances of uterine malignant mixed müllerian tumors. AJR Am J Roentgenol 2010;195:1268-1275

48. Genever AV, Abdi S. Can MRI predict the diagnosis of endometrial carcinosarcoma? Clin Radiol 2011;66:621-624

49. Tanaka Y0, Tsunoda H, Minami R, Yoshikawa H, Minami M. Carcinosarcoma of the uterus: MR findings. J Magn Reson Imaging 2008;28:434-439

50. Takeuchi M, Matsuzaki K, Harada M. Carcinosarcoma of the uterus: MRI findings including diffusion-weighted imaging and MR spectroscopy. Acta Radiol 2016;57:1277-1284

51. Kato $H$, Kanematsu $M$, Furui $T$, Imai $A$, Hirose $Y$, Kondo $H$, et al. Carcinosarcoma of the uterus: radiologicpathologic correlations with magnetic resonance imaging including diffusion-weighted imaging. Magn Reson Imaging 2008;26:1446-1450

52. Hernández Mateo P, Méndez Fernández R, Serrano Tamayo E. Uterine sarcoma vs adenocarcinoma: can MRI distinguish between them? Radiologia 2016;58:199-206

53. Huang YT, Chang CB, Yeh CJ, Lin G, Huang HJ, Wang CC, et al. Diagnostic accuracy of 3.0T diffusion-weighted MRI for patients with uterine carcinosarcoma: assessment of tumor extent and lymphatic metastasis. J Magn Reson Imaging 2018;48:622-631

54. Smith-Bindman R, Kerlikowske K, Feldstein VA, Subak L, Scheidler J, Segal M, et al. Endovaginal ultrasound to exclude endometrial cancer and other endometrial abnormalities. JAMA 1998;280:1510-1517

55. Felix AS, Weissfeld JL, Stone RA, Bowser R, Chivukula M, Edwards RP, et al. Factors associated with Type I and Type II endometrial cancer. Cancer Causes Control 2010;21:1851-1856

56. Amant F, Mirza MR, Koskas M, Creutzberg CL. Cancer of the corpus uteri. Int J Gynaecol Obstet 2015;131 Suppl 2:S96-S104

57. Creasman W. Revised FIG0 staging for carcinoma of the endometrium. Int J Gynaecol Obstet 2009;105:109

58. Kinkel K, Kaji Y, Yu KK, Segal MR, Lu Y, Powell CB, et al. Radiologic staging in patients with endometrial cancer: a meta-analysis. Radiology 1999;212:711-718

59. Fujii S, Kido A, Baba T, Fujimoto K, Daido S, Matsumura $\mathrm{N}$, et al. Subendometrial enhancement and peritumoral enhancement for assessing endometrial cancer on dynamic contrast enhanced MR imaging. Eur J Radiol 2015;84:581-589

60. Andreano A, Rechichi G, Rebora P, Sironi S, Valsecchi MG, Galimberti S. MR diffusion imaging for preoperative staging of myometrial invasion in patients with endometrial cancer: a systematic review and meta-analysis. Eur Radiol 2014;24:1327-1338

61. Ota T, Hori M, Onishi H, Sakane M, Tsuboyama T, Tatsumi M, et al. Preoperative staging of endometrial cancer using reduced field-of-view diffusion-weighted imaging: a preliminary study. Eur Radiol 2017;27:5225-5235

62. Takeuchi M, Matsuzaki K, Harada M. Evaluating myometrial invasion in endometrial cancer: comparison of reduced fieldof-view diffusion-weighted imaging and dynamic contrastenhanced MR imaging. Magn Reson Med Sci 2018;17:28-34 
63. Bhosale $P$, Ma J, Iyer R, Ramalingam $P$, Wei W, Soliman $P$, et al. Feasibility of a reduced field-of-view diffusion-weighted ( $\mathrm{FOV}$ ) sequence in assessment of myometrial invasion in patients with clinical FIG0 stage I endometrial cancer. J Magn Reson Imaging 2016;43:316-324

64. Eskander RN, Randall LM, Berman ML, Tewari KS, Disaia PJ, Bristow RE. Fertility preserving options in patients with gynecologic malignancies. Am J Obstet Gynecol 2011;205:103110

65. Lin G, Huang YT, Chao A, Ng KK, Yang LY, Ng SH, et al. Influence of menopausal status on diagnostic accuracy of myometrial invasion in endometrial cancer: diffusionweighted and dynamic contrast-enhanced MRI at 3 T. Clin Radiol 2015;70:1260-1268

66. Turan T, Hizlı D, Yilmaz SS, Gundogdu B, Boran N, Tulunay G, et al. What is the impact of cervical invasion on lymph node metastasis in patients with stage IIIC endometrial cancer? Arch Gynecol Obstet 2012;285:1119-1124

67. Haldorsen IS, Berg A, Werner HM, Magnussen IJ, Helland H, Salvesen 00, et al. Magnetic resonance imaging performs better than endocervical curettage for preoperative prediction of cervical stromal invasion in endometrial carcinomas. Gynecol Oncol 2012;126:413-418

68. Luomaranta A, Leminen A, Loukovaara M. Magnetic resonance imaging in the assessment of high-risk features of endometrial carcinoma: a meta-analysis. Int J Gynecol Cancer 2015;25:837-842

69. Freeman SJ, Aly AM, Kataoka MY, Addley HC, Reinhold C, Sala E. The revised FIGO staging system for uterine malignancies: implications for MR imaging. Radiographics 2012;32:1805-1827

70. Lin G, Huang YT, Chao A, Lin YC, Yang LY, Wu RC, et al. Endometrial cancer with cervical stromal invasion: diagnostic accuracy of diffusion-weighted and dynamic contrast enhanced MR imaging at 3T. Eur Radiol 2017;27:1867-1876

71. Tamai K, Koyama T, Saga T, Umeoka S, Mikami Y, Fujii S, et al. Diffusion-weighted MR imaging of uterine endometrial cancer. J Magn Reson Imaging 2007;26:682-687

72. Nougaret S, Reinhold C, Alsharif SS, Addley H, Arceneau J, Molinari $\mathrm{N}$, et al. Endometrial cancer: combined MR volumetry and diffusion-weighted imaging for assessment of myometrial and lymphovascular invasion and tumor grade. Radiology 2015;276:797-808

73. Cao K, Gao M, Sun YS, Li YL, Sun Y, Gao YN, et al. Apparent diffusion coefficient of diffusion weighted MRI in endometrial carcinoma-Relationship with local invasiveness. Eur J Radiol 2012;81:1926-1930

74. Shih IL, Yen RF, Chen CA, Chen BB, Wei SY, Chang WC, et al. Standardized uptake value and apparent diffusion coefficient of endometrial cancer evaluated with integrated whole-body PET/MR: correlation with pathological prognostic factors. J Magn Reson Imaging 2015;42:1723-1732

75. Zhang J, Cai S, Li C, Sun X, Han X, Yang C, et al. Can magnetic resonance spectroscopy differentiate endometrial cancer? Eur Radiol 2014;24:2552-2560
76. Han X, Kang J, Zhang J, Xiu J, Huang Z, Yang C, et al. Can the signal-to-noise ratio of choline in magnetic resonance spectroscopy reflect the aggressiveness of endometrial cancer? Acad Radiol 2015;22:453-459

77. Ueno Y, Forghani B, Forghani R, Dohan A, Zeng XZ, Chamming's $F$, et al. Endometrial carcinoma: MR imagingbased texture model for preoperative risk stratification-A preliminary analysis. Radiology 2017;284:748-757

78. Brown AP, Gaffney DK, Dodson MK, Soisson AP, Belnap TW, Alleman K, et al. Survival analysis of endometrial cancer patients with positive lymph nodes. Int J Gynecol Cancer 2013;23:861-868

79. Creasman WT, Odicino F, Maisonneuve P, Quinn MA, Beller U, Benedet JL, et al. Carcinoma of the corpus uteri. FIG0 26th annual report on the results of treatment in gynecological cancer. Int J Gynaecol Obstet 2006;95 Suppl 1:S105-S143

80. ASTEC study group, Kitchener H, Swart AM, Qian Q, Amos C, Parmar MK. Efficacy of systematic pelvic lymphadenectomy in endometrial cancer (MRC ASTEC trial): a randomised study. Lancet 2009;373:125-136

81. Benedetti Panici P, Basile S, Maneschi F, Alberto Lissoni A, Signorelli M, Scambia G, et al. Systematic pelvic lymphadenectomy vs. no lymphadenectomy in early-stage endometrial carcinoma: randomized clinical trial. J Natl Cancer Inst 2008;100:1707-1716

82. Todo Y, Kato H, Kaneuchi M, Watari H, Takeda M, Sakuragi N. Survival effect of para-aortic lymphadenectomy in endometrial cancer (SEPAL study): a retrospective cohort analysis. Lancet 2010;375:1165-1172

83. Eggemann H, Ignatov T, Kaiser K, Burger E, Costa SD, Ignatov A. Survival advantage of lymphadenectomy in endometrial cancer. J Cancer Res Clin Oncol 2016;142:1051-1060

84. Lin G, Ho KC, Wang JJ, Ng KK, Wai YY, Chen YT, et al. Detection of lymph node metastasis in cervical and uterine cancers by diffusion-weighted magnetic resonance imaging at 3T. J Magn Reson Imaging 2008;28:128-135

85. Park JY, Kim EN, Kim DY, Suh DS, Kim JH, Kim YM, et al. Comparison of the validity of magnetic resonance imaging and positron emission tomography/computed tomography in the preoperative evaluation of patients with uterine corpus cancer. Gynecol Oncol 2008;108:486-492

86. Lai CH, Lin G, Yen TC, Liu FY. Molecular imaging in the management of gynecologic malignancies. Gynecol Oncol 2014;135:156-162

87. Lee HJ, Park JY, Lee JJ, Kim MH, Kim DY, Suh DS, et al. Comparison of MRI and 18F-FDG PET/CT in the preoperative evaluation of uterine carcinosarcoma. Gynecol Oncol 2016;140:409-414

88. Stecco A, Buemi F, Cassarà A, Matheoud R, Sacchetti GM, Arnulfo A, et al. Comparison of retrospective PET and MRIDWI (PET/MRI-DWI) image fusion with PET/CT and MRI-DWI in detection of cervical and endometrial cancer lymph node metastases. Radiol Med 2016;121:537-545 